\title{
DISCRETE ANALOGUES OF SINGULAR RADON TRANSFORMS
}

\author{
E. M. STEIN AND S. WAINGER
}

The purpose of this paper is to describe recent results we have obtained in finding discrete analogues of the theory of singular integrals on curves, or more generally of "singular Radon transforms," at least in the translation-invariant case. Our theorems are related to estimates for certain exponential sums that arise in number theory; they are also connected with Bourgain's recent maximal ergodic theorem [2, 3]. The detailed proofs of our results are quite lengthy, and will appear elsewhere. Here we shall limit ourselves to stating the main conclusions, and sketching the motivation and background. We take this opportunity to acknowledge our indebtedness to Guido Weiss and A. De la Torre, whose suggestions were the starting point of this research.

The theory of singular Radon transforms may be thought of as a natural extension of the Calderon-Zygmund theory of singular integrals. It had as its origin some problems related to parabolic differential equations, and was developed further because of its realvariable consequences, in particular differentiation theory along lower-dimensional varieties; see [10]. Later its relevance to several complex variables and its connection with analysis on nilpotent Lie groups were brought out; (see $[6,7,8]$ ). Here we begin by stating one of the main known results in the $\mathbf{R}^{n}$ setting for a basic model problem.

Let $K$ be a Calderón-Zygmund convolution kernel on $\mathbf{R}^{k}$ (here $k$ need not equal $n$ ). Then $K$ is defined away from the origin, satisfies the estimates $|K(x)| \leq A|x|^{-k},|\nabla k(x)| \leq A|x|^{-k-1}$, and the cancellation property: $\int_{R \leq|x| \leq \gamma R} K(x) d x=0$, for some $\gamma>1$, and all $0<R<\infty$.

Received by the editors March 29, 1990.

1980 Mathematics Subject Classification (1985 Revision). Primary 42B20, 42B99, $11 \mathrm{~L} 40$. 
We also suppose we are given a polynomial map $P: \mathbf{R}^{k} \rightarrow \mathbf{R}^{n}$, i.e. $P=\left(P_{1}, P_{2}, \ldots, P_{n}\right)$, where the $P_{\ell}$ are real-valued polynomials on $\mathbf{R}^{k}$. We consider the transformation $T$, defined by

$$
T(f)(x)=\int_{\mathbf{R}^{k}} f(x-P(y)) K(y) d y
$$

which can be defined on the Schwartz space of $\mathbf{R}^{n}$ as a generalized principal-value. Then a restatement of the known results is as follows:

Theorem A. T extends to a bounded operator on $L^{p}\left(\mathbf{R}^{n}\right)$ to itself, $1<p<\infty$. Moreover the norm of $T$ has a bound which depends only on the degree of $P$ (and of course on $p$ and $K$ ), but is otherwise independent of $P$.

The discrete analogue of (1) can be formulated as follows. We consider the operator $S$, given by

$$
S(f)(x)=\sum_{j \in \mathbf{Z}^{k}}{ }^{\prime} f(x-P(j)) K(j),
$$

where $\mathbf{Z}^{k}$ denotes the integral lattice in $\mathbf{R}^{k}$, and the prime in (2) indicates that $j=0$ is excluded from the sum. The question we deal with is that of the bounds

$$
\|S(f)\|_{L^{p}\left(\mathbf{R}^{n}\right)} \leq A\|f\|_{L^{p}\left(\mathbf{R}^{n}\right)} .
$$

In the special case the polynomials map $\mathbf{Z}^{k}$ to $\mathbf{Z}^{n}$ (e.g. when they have integer coefficients) we can also consider the mapping $S$ as transforming functions on $\mathbf{Z}^{n}$ to functions on $\mathbf{Z}^{n}$. In this case we can also formulate the question whether

$$
\|S(f)\|_{\ell^{p}\left(\mathbf{Z}^{n}\right)} \leq A\|f\|_{\ell^{p}\left(\mathbf{Z}^{n}\right)} .
$$

Our main result is a partial analogue of the continuous case described by Theorem A. While the result below deals only with a range of $p$, it is not unreasonable to expect that ultimately it will be shown to hold for all $p, 1<p<\infty$.

Theorem 1. The operator $S$ is bounded on $L^{p}\left(\mathbf{R}^{n}\right)$ to itself for $3 / 2<p<3$. Moreover the norm of $S$ has a bound that depends only on the degree of $P$ (and $p$ and $K$ ), but is otherwise independent of $P$. In the case that $P$ maps $\mathbf{Z}^{k}$ to $\mathbf{Z}^{n}$, similar results hold for $3^{\prime}$. 
There are several variants of the above which are possible; here we state two. First, let $x \rightarrow U^{x}$ be a continuous mapping of $\mathbf{R}^{n}$ to unitary operators on some Hilbert space $\mathscr{H}$, which satisfies the multiplicative property: $U^{x} \cdot U^{y}=U^{x+y}, x, y \in \mathbf{R}^{n}$. Then the (appropriately defined) operator

$$
S_{1}=\sum_{j \in \mathbf{Z}^{k}}{ }^{\prime} U^{P(j)} K(j)
$$

is bounded on $\mathscr{H}$.

Further, suppose $(X, d \mu)$ is a $\sigma$-finite measure space, and each $U^{x}$ is realized as induced by a measure-preserving transformation of $(X, d \mu)$. Then the operator (4) is bounded on $L^{p}(X, d \mu)$ to itself, $3 / 2<p<3$. There are also variants when the mappings $x \rightarrow U^{x}$ are defined only for $x \in \mathbf{Z}^{n}$; then one requires that the polynomial $P$ maps $\mathbf{Z}^{k}$ to $\mathbf{Z}^{n}$.

Beyond the motivation of the continuous case described in $\S 1$, we mention here three other sources for our work - to which we shall return below.

First, the extensions of the maximal ergodic theorem due to Bourgain [2,3]. He proved in the setting when $U$ is induced by a measure-preserving transformation, that the maximal operator $f \rightarrow \sup _{N} \frac{1}{N}\left|\sum_{1 \leq j \leq N} U^{P(j)} f\right|$ is bounded on $L^{p}(X, d \mu)$ to itself, for $1<p<\infty$. Here $P=\left(P_{1}, P_{2}, \ldots, P_{n}\right)$ is a polynomial from $\mathbf{Z}^{1}$ to $\mathbf{Z}^{n}$. Bourgain used the techniques of the "circle method" of Hardy and Littlewood, as further developed by Vinogradov and others (see [11]), which divides the spectrum into "major" and "minor" arcs. We also use these techniques in an essential way, as indicated below.

Second, we showed in the case $P$ is a quadratic polynomial, then the result for $p=2$ is deducible from Carleson's theorem [4] on the convergence of Fourier series (in its $n$-dimensional form given in Sjölin [9]). We return to this in $\S 7$ below.

Third, is the work of Arkhipov and Oskolkov [1]. They proved, in effect, the case corresponding to $p=2$, and in one dimension (i.e., $k=1$ ), also using the circle method. Unfortunately we did not know their results when we undertook our work; their paper had been overlooked by us, and others*.

*Only in March 1989, after one us lectured on our work was their paper brought to our attention. 
We now describe some other consequences of our methods of proof. These involve the space of functions of bounded mean oscillation.

Theorem 2. Suppose $d$ is a positive integer. Then the function $f(\theta)=\sum_{j=1}^{\infty} e^{i j^{d} \theta} / j$ is in BMO.

We also state two variants of this result. Let $P_{1}, \ldots, P_{n}$ be $n$ polynomials on $\mathbf{R}^{1}$, each with integer coefficients. For $\theta=$ $\left(\theta_{1}, \ldots, \theta_{n}\right) \in T^{n}$, write $\theta \cdot P(j)$ for $\theta_{1} P_{1}\left(j_{1}\right)+\theta_{2} P_{2}\left(j_{2}\right)+\cdots+$ $\theta_{n} P_{n}\left(j_{n}\right)$ where $j=\left(j_{1}, \ldots, j_{n}\right) \in \mathbf{Z}^{n}$.

Theorem 3. The function $f(\theta)=\sum_{j \in \mathbf{Z}^{n}}^{\prime}\left(e^{i \theta \cdot P(j)} /|j|^{n}\right)$ is in $B M O\left(T^{n}\right)$.

Next, let $r_{k, d}(j)$ equal the number of representations of the integer $j$ as a sum of $k, d$ th powers, which is the counting function in Waring's problem.

Theorem 4. The function $\sum_{j=1}^{\infty} r_{k, d}(j)\left(e^{i j \theta} / j^{k / d}\right)$ is in $B M O\left(T^{1}\right)$.

One can make more general statements of this kind if one also uses an observation we owe to J. L. Journé: Namely, if $\sum a_{k} e^{i k \theta}$ with $a_{k} \geq 0$ is in $B M O\left(T^{1}\right)$, then so is $\sum b_{k} e^{i k \theta}$ wherever $\left|b_{k}\right| \leq$ $a_{k}$.

5

A natural problem raised by the theory of the continuous case and our results is the extension of the above when $\mathbf{R}^{n}$ is replaced by a more general nilpotent group (and $\mathbf{Z}^{n}$ is replaced by a corresponding discrete subgroup). That it is reasonable that substantial results along these lines are to be expected is indicated by the following, which we have obtained for the Heisenberg group. Let $H^{1}=\left\{\left(x_{1}, x_{2}, x_{3}\right)\right\}$ be the Heisenberg group with multiplication $x \cdot y=\left(x_{1}+y_{1}, x_{2}+y_{2}, x_{3}+y_{3}+x_{1} y_{2}-x_{2} y_{1}\right)$. The points $\mathbf{Z}^{3}$ form a subgroup. Suppose $K$ is a Calderón-Zygmund kernel on $\mathbf{R}^{2}$ and let $(T f)(x)=\sum_{j \in \mathbf{Z}^{2}}^{\prime} f\left(x \cdot\left(j_{1}, j_{2}, 0\right)\right) K(j)$.

Theorem 5. The mapping $f \rightarrow T(f)$ is bounded on $L^{2}\left(H^{1}\right)$ to itself. 
By similar methods one can also prove that the maximal operator

$$
f \rightarrow \sup _{R>0} \frac{1}{R^{2}}\left|\sum_{j_{1}^{2}+j_{2}^{2} \leq R^{2}} f\left(x \cdot\left(j_{1}, j_{2}, 0\right)\right)\right|
$$

is bounded on $L^{p}\left(H^{1}\right), 2 \leq p$. The results are discrete analogues of operators considered in $[6,7]$.

6

We indicate the method of proof of Theorem 1 for $p=2$. For the continuous analogue given by (1), the $L^{2}$ boundedness reduced to the following estimate

$$
\left|\int_{\mathbf{R}^{k}} e^{i Q(y)} K(y) d y\right| \leq A
$$

where $Q$ is any real polynomial on $\mathbf{R}^{k}$, and the bound $A$ depends only on the degree of $Q$ (and of course on $K$ ). For the discrete analogue there is an analogous result, which is of interest in its own right.

\section{Lemma.}

$$
\left|\sum_{\substack{j \in \mathbf{Z}^{k} \\|j| \leq R}} e^{2 \pi i Q(j)} K(j)\right| \leq A .
$$

Here again the bound depends only on the degree of $Q$ and on $K$, but not on $R$. The proof of (5) proceeded by exploiting the method of stationary phase, and dilations on $\mathbf{R}^{k}$. However (6) is subtler, since neither ideas are applicable; it requires the circle method. More precisely we write $Q(y)=\sum_{|\alpha| \leq d} \theta_{\alpha} y^{\alpha}$, where $d$ is the degree of $Q$. We then decompose the full sum in (6) into dyadic parts $\sum_{2^{\prime} \leq|j| \leq 2^{\ell+1}} e^{2 \pi i Q(j)} K(j)$, and try to control each part. Now we divide the $\theta$ space into "major" and "minor" boxes, following the philosophy of Hardy and Littlewood. The former consist of those $\theta$ where the $\theta_{\alpha}$ are approximately close to rationals with small denominators; and the latter consist of the complementary $\theta^{\prime} s$. The latter contributions are "small," 
and are estimated by reducing matters to estimates for Weyl sums. The former contain the main contribution. These can be replaced (again making small errors) by products involving the appropriate generalized Gauss sums (see (7) below), and the corresponding contributions form the continuous case, which is the integral (5).

The generalized Gauss sums can be written as follows: $P$ is a polynomial of $k$ variables of degree $d$, with integer coefficients which are relatively prime to a "denominator" $q$. We then consider

$$
S_{q}=\sum_{j} \exp (2 \pi i P(j) / q)
$$

where the sum is taken over the range $1 \leq j_{1} \leq q, 1 \leq j_{2} \leq$ $q, \ldots 1 \leq j_{k} \leq q$. The estimate we need is then that for some $\varepsilon=\varepsilon_{d}, \varepsilon_{d}>0$,

$$
\left|S_{q}\right| \leq A_{d} q^{k-\varepsilon}
$$

Below we shall also need the following estimate. Let $m(q)$ denote the maximum of the absolute values of all sums in (7) with denominator $q$. For $0<\alpha<1$, let $N(\alpha)$ denote the number of $q$ 's so that $q^{-k} m(q)>\alpha$. Then

$$
N(\alpha)=O\left(\alpha^{-2-\varepsilon}\right) \quad \text { as } \alpha \rightarrow 0, \quad \text { for every } \varepsilon>0 .
$$

The estimate (8) is obtained by reducing matters to estimates for Weyl sums. For (9) one uses induction, the multiplicative properties of sums (7), and ultimately Weil's estimates when $q$ is a prime in the one-dimensional case. See also Carlitz-Uchiyama [5] and Bourgain [3] for some related statements.

7

We now point out an alternative proof of the main lemma above in the case when $Q$ is at most degree 2 , without using the circle method. It is based on the following.

Lemma. The operator $f \rightarrow \sup _{\xi \in \mathbf{R}^{k}}\left|\sum_{j \in \mathbf{Z}^{k}}^{\prime} e^{i \xi \cdot j} K(j) f(x-j)\right|$ is bounded on $L^{2}\left(\mathbf{Z}^{k}\right)$ to itself.

This is a discrete version of Sjölin's generalization of Carleson's theorem. The case $d=2$ of the main lemma follows from it if we apply the above to $f(x) e^{i Q_{0}(x)}$, where $Q_{0}$ is an arbitrary homogeneous polynomial of degree 2 , and where we choose $\xi=$ 
$\left(\nabla Q_{0}\right)(x)$. As a consequence, the operator

$$
f \rightarrow \sum_{j}^{\prime} e^{i Q_{0}(j)} K(j) f(x-j)
$$

is bounded on $L^{2}$, with a bound independent of $Q_{0}$, from which the desired result follows.

\section{8}

The proof of Theorem 1 , when $p \neq 2$ follows the idea of the argument given in Bourgain [3], for the proof of his maximal ergodic theorem cited in $\S 3$. Here we will only indicate how the limitation $3 / 2<p<3$ comes about. In considering the major boxes which arose in $\S 5$, we further group these contributions according to the sizes for the sums (7). Now the norm on $L^{2}$ of an appropriate subsum is majorized by $O\left(2^{-m}\right)$. (The subsum corresponds to those $q$ 's, where $\left.q^{-k} m(q) \approx 2^{-m}\right)$. On the other hand, one can show that the $L^{p}$ norm of such a subsum is dominated by $O\left(2^{(2+\varepsilon) m}\right)$, using essentially Theorem A, for $1<p<\infty$, and estimate (9). An interpolation then gives the $L^{p}$ bound $O\left(2^{-\varepsilon^{\prime} m}\right)$, wherever $3 / 2<p<3$, and the convergence of the series $\sum_{m>0} 2^{-\varepsilon^{\prime} m}$ allows us to obtain the $L^{p}$ boundedness of the operator $S$ in (2).

\section{REFERENCES}

1. G. I. Arkhipov and K. I. Oskolkov, On a special trigonometric series and its applications, Mat. Sb. 134(176) (1987), 147-158; Soviet Math 62 (1989), 145-156.

2. J. Bourgain, On the maximal ergodic theorem for certain subsets of integers, Israel J. Math. 61 (1988), 39-72; 73-83.

3. __ Return times of dynamical systems, Inst. Hautes Études Sci. preprint.

4. L. Carleson, On the convergence and growth of partial sums of Fourier series, Acta Math. 116 (1966), 135-157.

5. L. Carlitz and S. Uchiyama, Bounds for exponential sums, Duke Math. J. 24 (1957), 37-41.

6. D. Geller and E. M. Stein, Estimates for singular convolution operators on the Heisenberg group, Math. Ann. 267 (1984), 1-15.

7. D. H. Phong and E. M. Stein, Hilbert integrals, singular integrals, and Radon transforms I, Acta Math. 157 (1986), 99-757.

8. F. Ricci and E. M. Stein, Harmonic analysis on nilpotent groups and singular integrals, J. Funct. Anal. 73 (1987), 179-194; also 78 (1988), 56-84.

9. P. Sjölin, Convergence almost everywhere of certain singular integrals and multiple Fourier series, Ark. Mat. 9 (1971), 65-90. 
10. E. M. Stein and S. Wainger, Problems in harmonic analysis related to curvature, Bull. Amer. Math. Soc. 84 (1978), 1239-1295.

11. I. Vinogradov, The method of trigonometrical sums in the theory of numbers, Interscience, New York, 1954.

Princeton University, Princeton, New Jersey 08544

UNIVERSITY OF WiSCONSIN, MADISON, Wisconsin 53706 